\title{
Un wiki sobre Amor y pedagogía, de Miguel de Unamuno
}

\author{
Inmaculada López \\ Universidad Autónoma de Barcelona, España
}

\section{Introducción}

El presente trabajo tiene como motivo el estudio de un wiki que el alumnado de $2^{\circ}$ de bachillerato realizó durante el curso 2009-2010 y cuya dirección web es: http://amorypedagogia.wikispaces.com/. Se trataba de estudiar en clase de Lengua y Literatura Castellanas una obra de la que apenas había bibliografía adecuada para los estudiantes -Amor y pedagogía, de Miguel de Unamuno- y poner más tarde por escrito lo aprendido. El objetivo principal del trabajo es comprobar los beneficios de la escritura colaborativa en entornos tecnológicos, $y$, asociada a esta escritura, los recursos que esta tecnología ofrece a una metodología de trabajo de colaboración: la interacción en el seno de una comunidad de prácticas conformada por los estudiantes. Los resultados de la experiencia se consideraron positivos, pero quedó pendiente estudiar por qué lo fueron y rescatar los elementos más pobres de la tarea para, en la medida de lo posible, volver a plantearla en cursos sucesivos y cuando los grupos y los temas lo permitieran. La investigación contribuiría a ello.

Las herramientas tecnológicas pueden utilizarse en el aula para ampliar las estrategias de enseñanza - aprendizaje, pero también para familiarizar a los estudiantes con unos recursos que seguramente tendrán mucha importancia para ellos en el futuro. El poner por escrito, sistematizándolos, los elementos que intervinieron en esas estrategias podían dar idea de las virtudes y los defectos, de las luces y las sombras del procedimiento, de la herramienta y del proceso que se siguió en su momento. Para ello, se dividió el estudio en diversos apartados, que son los mismos que expondremos a continuación: en primer lugar, unas reflexiones teóricas sobre lo que implica la escritura colaborativa con recursos TIC que fundamentan su utilización. En segundo lugar, la descripción de la actividad, de la secuencia didáctica que se realizó en el aula. La parte práctica o secuencia de la escritura recoge la preparación, la realización y la evaluación del texto. Finalmente, se sintetizan los resultados de la actividad en dos aspectos 
fundamentales: la función que tuvo el foro de discusión y las dificultades y aciertos del trabajo colaborativo en el wiki.

\section{Marco teórico}

En este apartado se presentan dos conceptos diferenciados que se deben tener en cuenta. Por una parte, el objetivo de que el alumnado participa en tareas colaborativas de aprendizaje e interacciona en busca de acuerdos y de enriquecimiento cognitivo. Por otra, la utilización de una herramienta que permite visualizar rápidamente el proceso de esa interacción entre ellos mismos pero también a la profesora, quien tendrá que evaluar ese proceso de construcción de conocimientos y la implicación y motivación personal en la tarea de cada componente de los grupos de trabajo. El wiki permitía abarcar ambos requerimientos de la tarea. Intentaremos definir mejor esos presupuestos.

Para empezar, entendemos que la escritura en este entorno tendría una doble función: una función representativa, por cuanto el alumnado escribe para mostrar unos conocimientos, explicarlos y hacerlos accesibles a otras personas; y otra función comunicativa, porque escribir implica tener que interactuar con otros individuos que contribuyen también con su saber al texto conjunto, y se necesita un acuerdo que permita coordinarse y, muchas veces, convencer de una propuesta concreta. De esta manera, la escritura es una escritura reflexiva por lo que tiene de toma de conciencia de unos saberes, de construcción del pensamiento individual y colectivo, pero también de autorregulación individual y colectiva. El alumnado que escribe colaborativamente necesita representarse primeramente sus conocimientos de forma individual, reflexionarlos e intentar aproximarse en borradores previos. Después ya puede exponerlos públicamente para ser debatidos. Esto implica:

- La capacidad de reconocer que todos los textos son revisables y perfeccionables, de cuestionarse el propio texto y el texto de los otros.

- La capacidad de admitir que otro intervenga en el texto propio para modificarlo, o de intervenir en el texto de otros para mejorarlo.

Son varios los conceptos que debemos tener en cuenta para desarrollar este tema. Aprendizaje compartido en una comunidad de prácticas: Desde Lave y Wenger (1991) se ha investigado mucho sobre lo que implica colaborar entre iguales para realizar un aprendizaje situado y significativo. Estos autores distinguen tres condicionantes: el dominio, la comunidad y la práctica. Faltaría un elemento cohesionador y necesario en las comunidades escolares: la figura del docente que guía el proceso de construcción del 
conocimiento desde su misma planificación. Así, los estudiantes están unidos por un objetivo común y comparten un conocimiento más o menos homogéneo del tema que deben desarrollar; no son profesionales en el sentido estricto de la palabra, pero se entrenan para serlo y comparten sus experiencias, conocimientos y estrategias de aprendizaje. Y son comunidad de prácticas en una doble vertiente: los grupos pequeños desarrollan un mismo concepto y lo escriben, pero a la vez esos pequeños grupos que han desarrollado temas diferentes pertenecen a una comunidad mayor en la que se suman y se coordinan todos los conceptos, se revisan y los valoran, alcanzando así sentido pleno.

Interacción y discusión en grupo: Olga Esteve (2002), basándose en las ideas de Vigotsky (1995), defiende la concepción socioconstructivista del aprendizaje a través de la interacción. El aula no sólo es un espacio de conocimiento sino también un espacio social y comunicativo que permite compartir conocimientos con el fin de aprender. Para ello el docente ha de procurar partir de la Zona de Desarrollo Próximo de que habla Vigotsky (1995) -aquello que cada alumno y alumna ya sabe- para levantar la coconstrucción del conocimiento en la interacción con los otros. El debate oral en clase, una vez se han puesto de manifiesto los puntos de partida del colectivo, potencia la reflexión conjunta y se aclaran conceptos que después se explicitan en el trabajo por tareas. Esa toma de conciencia del propio aprendizaje posibilitará la autorregulación del alumnado, tanto individual como colectivo.

En la tarea que se propone es vital la interacción oral y escrita de los participantes de los diferentes grupos. Con ella consiguen: coordinarse en las labores que han de desarrollar; valorar lo que ponen los compañeros y compañeras del equipo y, por lo tanto, ofrecer la mejor visión crítica, ya que del buen trabajo parcial depende el éxito de todos; aprender a negociar y a argumentar sobre contenidos y estrategias. En cuanto a la escritura, si bien es siempre una pluma la que empieza escribiendo un texto, son todos los demás participantes del pequeño grupo y posteriormente los del grupo total los que revisan, proponen y modifican ese mismo texto. La parte más importante, por lo tanto, de la interacción en la actividad que describimos es la de la revisión textual.

Conocimiento mediado por ordenador: Scardamaglia y Bereiter (1994) ya nos hablaban de este concepto. Hablan de la escuela como comunidades de construcción o edificación del conocimiento (knowledge-building communities). Entienden esta edificación del conocimiento como una actividad colaborativa orientada al desarrollo de 
dispositivos conceptuales y al perfeccionamiento de la comprensión colectiva, a partir de la tecnología como facilitadora de esas comunidades de prácticas que ya hemos visto, porque la proyectan más allá de sus paredes y la conectan con el mundo, con otras comunidades, de forma natural.

Si para Bustos (2009) la tecnología nos permite hacer un seguimiento exhaustivo de los procesos, tremendamente válido para la evaluación formativa y formadora, la tesis que sustentamos es que lo mismo ocurrirá con los procesos de escritura colaborativa a través de herramientas tecnológicas. Advierte, no obstante, Bustos que a diferencia de la escritura individual, la escritura colaborativa contiene un proceso que consiste en seleccionar las ideas que se quiere escribir, estructurar el texto, decidir los contenidos y revisar varias veces antes de entregar el texto definitivo. Además, la comunicación que llevan a cabo los grupos tiene un cierto carácter argumentativo por cuanto los participantes negocian "propósitos, planes, conceptos y dudas”. Deben estar coordinados y discutir. ¿Cómo? Esta coordinación se establece a partir de tres posibles vías:

- Cara a cara, cuando los participantes se ven y pueden manifestarse directamente sus dudas.

- A través de la comunicación síncrona como el chat.

- A través de la comunicación asíncrona, como los foros o el correo electrónico.

Las tres son necesarias en un momento u otro del proceso de escritura. Las tecnologías colaborativas nos permiten, en este sentido, una nueva forma de colaboración, modifican nuestra manera de trabajar y de relacionarnos entre nosotros y en Internet. El o la wiki es una tecnología colaborativa que permite tres acciones básicas (Coll 2005):

- Reflejar qué hacen y qué piensan los participantes: capturar interacciones.

- Mostrar las relaciones entre los participantes, y entre los participantes y los recursos que utilizan.

- Permitir a los participantes actuar libremente en sus motivaciones, deseos y actitudes: preservar la autonomía y la independencia.

El wiki dispone de un foro de discusión que refleja los intercambios que los participantes realizan sobre los contenidos y que son variadísimos. Igualmente, para el docente es especialmente útil el historial de cambios que se han producido en una misma página, ya que consigna la persona que los realiza y el cambio que propone. Se trata de una ayuda inestimable para la evaluación formativa y la sumativa. 
Paredes et al. (2005) afirman que en el wiki coexisten dos agentes participantes: docente y alumnado. El papel del docente es doble, ya que interviene activamente en el pre-proceso de escritura y en la fase de post-proceso de escritura (ayuda en la revisión y en la edición y evalúa). En la primera, que es la fase de planificación y preparación, define la actividad que han de realizar los estudiantes y planifica cómo se va a desarrollar; además, diseña la página web donde se van a verter los contenidos. En la segunda, que coincide con la fase de revisión y evaluación, colabora en la revisión final, analiza y evalúa el producto final. En cuanto a los estudiantes, participan de forma muy activa en el proceso de aprendizaje, sabiendo que forman parte de una comunidad que tiene un objetivo común; sus actividades tienen un carácter cooperativo, para lo que necesitan establecer unas relaciones complejas en el seno de los componentes de esa comunidad.

Para que todo ello sea posible y no haya interferencias de fuera, ya que el wiki es un entorno abierto, el docente debe asegurarse de que mientras dura el proceso de escritura, la tarea que se ha planteado realizar con sus estudiantes, no habrá otros usuarios del wiki más que sus propios estudiantes. Una vez publicada la página web definitiva, puede abrirlo a más colaboraciones si se desea.

\section{Marco metodológico}

Se trata de una investigación-acción, ya que la misma investigadora es la profesora de aula. Los datos que se recogen y los resultados que se pretenden alcanzar con su análisis son los siguientes:

Cuadro 1: Datos recogidos y sus objetivos

\begin{tabular}{|l|l|}
\hline \multicolumn{1}{|c|}{ Datos } & \multicolumn{1}{c|}{ Objetivos } \\
\hline Historial del wiki & $\begin{array}{l}\text { Comprobar cómo se produce la construcción de conocimientos a través } \\
\text { de las sucesivas modificaciones que se realizan en la escritura }\end{array}$ \\
\hline Foro del wiki & $\begin{array}{l}\text { Comprobar que la interacción contribuye a la construcción del } \\
\text { conocimiento conjunto, pero también a la estrategia adoptada en el aula }\end{array}$ \\
\hline $\begin{array}{l}\text { Hojas de autoevaluación y de } \\
\text { evaluación mutua }\end{array}$ & $\begin{array}{l}\text { Detectar la visión del alumnado en cuanto a la idoneidad de la } \\
\text { metodología respecto del objetivo que se había fijado en la actividad. } \\
\text { Constituyen el elemento de triangulación para la validez del trabajo }\end{array}$ \\
\hline
\end{tabular}

La actividad se llevó a cabo con 55 alumnos y alumnas pertenecientes a dos grupos diferentes de bachillerato. La secuencia que se describirá a continuación se realizó de manera paralela en ambos grupos.

Además del objetivo prioritario expuesto en la introducción, al ser una investigación-acción, se pretende que las conclusiones del análisis sirvan como 
propuestas de mejora en sucesivas utilizaciones de esta herramienta TIC en situaciones de escritura colaborativa en el aula.

\section{Descripción de la actividad}

La actividad o secuencia didáctica se dividió en tres partes: la lectura y comentario en clase de la obra literaria, la redacción del wiki y su revisión.

La lectura de Amor y pedagogía: Tras una brevísima presentación de quién era el autor y los rasgos principales de la obra, especialmente en lo que a su estructura convenía, la profesora inició la lectura del primer capítulo en clase, abriendo un debate tras la lectura sobre diversos aspectos que el alumnado desconocía o que no había comprendido. Se les dio a continuación una guía de lectura y cada día, durante cuatro sesiones, debían traer leída y comentada una parte del libro. No se les pedía que el comentario lo escribieran, sino que lo trajeran simplemente reflexionado, porque en clase "hablaríamos" sobre lo que la lectura hubiera suscitado.

Se aprovechaba la lectura y el comentario para adentrarnos en otros aspectos relacionados con la obra o con el autor: biografía, ideas filosóficas, contexto sociopolítico... Los alumnos y alumnas tomaban notas y completaban lo que consideraban oportuno. Ya sabían que elaborarían un wiki cuyo principal interés era completar el conocimiento de la lectura que estaban haciendo, con lo que se les dejó muy claro que cuanta más información fueran recogiendo de la lectura, con más seguridad se enfrentarían al trabajo escrito.

La redacción del wiki: La profesora abrió un wiki en Wikispaces y dio de alta a los 55 alumnos y alumnas de los dos grupos de bachillerato. Veintisiete de ellos no pudieron acceder nunca por problemas técnicos. Sí se aseguró que como mínimo una persona de cada grupo tuviera su clave de acceso y pudiera editar en el wiki. En ocasiones, algunos alumnos entraban con el nombre de usuario de otros compañeros, firmando en el foro con su nombre real para ser reconocidos.

Los temas y la estructura se dieron a conocer tanto en el wiki como en el aula virtual que se utilizaba para el trabajo ordinario de aula. Se pretendía elaborar un texto complejo en el que se diera noticia de una obra muy escasamente estudiada hasta la fecha. Un imprescindible de la tarea era que, fuera cual fuera el tema que les correspondiera desarrollar, deberían ilustrarlo con citas o ejemplos del propio libro; sólo así se podría dar sentido al estudio. 
Si todo salía bien, la intención era realizar un enlace a la Wikipedia, en la que apenas había nada sobre la obra, y a alguna web educativa. Esto les hizo una especial ilusión y fue el acicate para que se esforzaran en escribir de manera personal, ya que redundaba en interés de todos el hecho de que fuera un estudio personal y no de 'copia y pega'.

Además, el mismo alumnado contestaba una hoja de autoevaluación y evaluación mutua que se les dio antes de acabar la revisión final.

La revisión de la escritura: La revisión formal contiene dos partes bien diferenciadas según el agente que la realiza:

1. El agente de la revisión es el mismo grupo. La revisión se realiza al mismo tiempo que se redacta el texto, si algún componente del grupo detecta un error de contenido o de forma, o al final de la redacción y antes de la ampliación de los hipervínculos, en cuyo caso siempre se encarga a un miembro concreto del equipo de trabajo que sea más ducho en las artes de la expresión escrita.

2. El agente de la revisión es el grupo clase con la mediación de la profesora. Fue la revisión más provechosa. Cuando todos los grupos hubieron dado por cerradas sus páginas respectivas, se dedicó un par de sesiones de clase a “corregir” todo aquello que se detectaba como error o que era mejorable. Como se trataba de dos grupos clase, se consensuó lo que cada uno corregiría atendiendo al criterio siguiente: se haría según la participación mayoritaria en el contenido de los componentes de ese grupo clase.

Más adelante se analizarán los resultados.

\section{Análisis de la secuencia de escritura}

Puesto que el objetivo principal de este trabajo es comprobar cómo se realiza la construcción conjunta de conocimientos a partir de una secuencia escrita sirviéndose de la tecnología, es preciso analizar la secuencia llevada a cabo para comprender los resultados obtenidos.

Como en la mayor parte de los procesos de escritura, también este estudio de la obra de Unamuno se realizó según los parámetros de planificación, textualización y revisión de que habla Camps (1994). La preparación de la actividad y de los condicionantes de la escritura corrió a cargo de la profesora mayoritariamente, al dar las directrices de trabajo. A lo largo del proceso, siguió la evolución de los grupos e intervino puntualmente en los foros de discusión y, finalmente, les ayudó en la revisión 
final. A continuación se detalla el proceso de trabajo y el análisis de cada una de las fases.

Fase de planificación: La parte de planificación tiene dos vertientes. Por un lado está la parte de contenido, en la cual los alumnos y alumnas han de dar estructurar su trabajo y recoger todo aquello que se dijo en clase para aportarlo al wiki. Por otra, tenemos la parte técnica, en que los estudiantes deben familiarizarse con la nueva herramienta y saber utilizarla.

En cuanto a los contenidos, partió de la profesora la planificación de los mismos y la estructura que tendría el wiki y, por tanto, el trabajo global. También diseñó la página web y envió las invitaciones a su alumnado. La razón por la que no se dejó que intervinieran en la estructura general fue que habían de participar dos clases diferentes y no era posible reunirlos a todos a la vez. Ellos y ellas se agruparon libremente según sus propias preferencias y escogieron el tema que después desarrollarían. En alguna ocasión fue la misma profesora la que propuso o impuso el tema cuando no quedó más remedio porque quedaran libres sin que nadie los hubiera escogido antes o por la dificultad o facilidad de los mismos que convenían a algunos estudiantes concretos. Por ejemplo, hay un alumnado especialmente ducho en sintetizar lo que dicen los demás; a éstos se les encargó que, una vez estuvieran las subsecciones pergeñadas, editaran las introducciones pertinentes. O a algún estudiante con dificultades en abstracción les encargó de la parte más mecánica, como el resumen de lo que se proponía el estudio de la obra. El wiki permite, en este aspecto, el tratamiento de la diversidad adecuado a cada tipo de alumnado, además del propio inherente al trabajo colaborativo.

En los primeros momentos también se explicaron los contenidos que deberían ir asociados a cada sección. Las dudas más importantes surgieron en la estructuración de cada uno de los temas. Por ejemplo, en los personajes. A pesar de que ya en clase habíamos hablado de los tres grupos de personajes que había en Amor y pedagogía, les quedó pendiente el de las mujeres y su concepción de la educación, opuesta a la del protagonista. La profesora hubo de recordarles en un par de ocasiones que lo recogieran. Además, añadieron el grupo de “personajes espontáneos” que después modificaron porque no era pertinente en esta obra: hubo de explicarles la diferencia entre personajes de teatro y personajes de novela.

En cuanto a la parte técnica del wiki, consignamos otra dificultad de la preparación. A pesar de que se les dio todo más o menos estructurado en función del tipo de comentario de la obra que se realizó en clase, se les aconsejó encarecidamente 
que hicieran pequeñas incursiones en esta herramienta en línea, que intentaran editar pruebas, para evitar posibles problemas técnicos de la web o de los usuarios y contraseñas. Aquí siempre hay un problema: los estudiantes no acostumbran, en líneas generales, a prever, esperan hasta el momento en que tienen contenidos a editar para utilizar la herramienta. Es entonces cuando se aperciben de los impedimentos y las dificultades, de manera que gran parte de las primeras semanas nos la pasamos solventando claves y permisos, y volviendo a recordar cómo se editaba en el wiki.

Fase del proceso de textualización: En un wiki es especialmente interesante comprobar día a día los cambios que se van haciendo en los textos. En la historia de cada página crecían las aportaciones y las correcciones. Destacarían de manera especial los siguientes aspectos:

La metodología: El grupo que trabajaba la sesión de Personajes estaba compuesto por once alumnos de tres grupos de trabajo diferentes pertenecientes a dos clases distintas. Los grupos que pertenecen a la misma clase tienen más fácil quedar un momento y ponerse de acuerdo en algún punto del contenido, pero no ocurre así cuando las clases no son las mismas, así que idearon un sistema de votaciones. Se trataba de que cada grupo editara una versión del contenido, la publicara en el wiki y, en el foro de discusión, votaran la que les parecía más acertada. Pero no se limitaron a votar versiones, sino que dividieron el contenido en partes que votaron independientemente. En algún caso, votaron personaje por personaje, sin atender a la categorización superior de principales y secundarios. Y no se limitaron a votar, sino que propusieron cambios sobre las versiones que habían recibido más votos. Este método fue adoptado poco más tarde por el grupo de Temas. Los dos grupos que intervienen en este foro pertenecían a clases diferentes. No les cuesta en absoluto llegar a acuerdos, porque la base metodológica se la habían facilitado los grupos que trabajaban Personajes. Eso sí, tuvieron especial cuidado en que todos los grupos se vieran reflejados en la participación.

La metodología de trabajo de estos grupos no está relacionada directamente con el proceso de escritura, pero es interesante ver cómo se organizan los estudiantes cuando han de realizar trabajos escritos en grupo. Cuando existen dos versiones o más del mismo tema, para ellos es un auténtico problema seleccionar la que consideran más válida porque no quieren herir susceptibilidades individuales. ¿Cómo decirle a alguien que lo que ha hecho no es suficientemente bueno para todo el colectivo que trabaja en lo mismo? En un principio, priman una visión democrática de entendimiento personal más 
que la calidad del propio escrito, aunque finalmente acabarán mejorando las versiones más pobres en el proceso final. Si hablamos de escritura colaborativa, hemos de tener en cuenta estos intentos de ponerse de acuerdo, esta metodología de trabajo en grupo (en grupos, podríamos decir) porque condicionarán la textualización y la revisión. Se trata de una estrategia de la escritura colaborativa.

Contraste de versiones: Relacionado con el punto anterior, tendríamos que remarcar una nueva función que otorgan al foro: la de contraste de versiones. Cuando los estudiantes barajan dos o tres versiones diferentes, llega un momento en que en la página del wiki publican la que consideran plausible o mejor, pero mantienen las otras, bien en un documento Word que uno de los participantes guarda en su PC, bien en el mismo foro, para que los otros compañeros puedan calibrar la pertinencia de los cambios.

La interacción en el proceso: Es también una cuestión interesante la interacción llevada a cabo por dos grupos determinados: el que trabaja El Positivismo y el que trabaja los Temas utilizan como herramienta paralela el chat. Frente a la comunicación asíncrona del foro de discusión del wiki, la comunicación sincrónica del chat, más inmediato, que les ayuda a quedar rápidamente de acuerdo. La negociación se establece de dos formas complementarias. Nadie les sugirió esta posibilidad del chat; igual que en el punto anterior, el uso surge de una necesidad a la que se busca una solución perfectamente satisfactoria para los participantes. Los otros grupos se limitan a quedar en clase y hablar personalmente de las dudas suscitadas en el foro. Quizá sea casualidad, pero los grupos que utilizaron el chat como herramienta complementaria no tuvieron problemas de coordinación y el entendimiento fue total.

Una de las propuestas de mejora para esta actividad es justamente la utilización de una herramienta de comunicación sincrónica, tal como propone Bustos (2009). Tiene las siguientes ventajas: es conocida por los participantes, ya que la utilizan en su vida cotidiana, permite una comunicación rápida y efectiva, también el envío de textos literales para la valoración inmediata, además de facilitar el contacto entre personas que no tienen posibilidad de hablar presencialmente de forma fácil. Esta herramienta puede ser el chat, pero también la videoconferencia con soporte textual.

El orden que siguen a la hora de editar en el wiki se refleja en la participación del foro. Por ejemplo, el grupo de los Temas, actúa de la siguiente manera:

1. Cada grupo redacta su versión del tema.

2. Publican todas las versiones. 
3. Votan las versiones completas o parciales que les parecen más acertadas.

4. Modifican algún aspecto de las versiones que han votado mayoritariamente.

5. Dan el formato adecuado (tipografía, espacios)

6. Añaden los hipervínculos internos (al propio wiki) y externos (generalmente, a la Wikipedia)

Una de las características que definen la escritura en Internet es el hipertexto que nace a partir del hipervínculo. Con él, la lectura deja de ser lineal porque sugiere nuevas vías de exploración. Para el lector inexperto puede suponer una distracción importante del objetivo que se haya marcado en un comienzo, pero para el lector experto es una posibilidad de ampliar el espectro de información que puede recabar. En cuanto a la escritura, la utilización del hipervínculo por parte del alumnado suele ser un elemento extraño, en tanto que está acostumbrado a la confección de un texto expositivo lineal. Se trata de un "añadido” que se realiza al final y que en prácticamente todas las ocasiones tuvo que recordar la profesora. La escritura en el wiki ya es compleja en sí misma, en gran parte por lo que tiene de negociación de contenidos, de expresión y de formato, pero se hace aún más dificultosa cuando se tiene que pensar en los elementos del texto susceptibles de implementar significados. El hecho de que la propuesta inicial de textualizar el contenido en el formato característico del reportaje periodístico fallara, se debió en gran parte a dos dificultades:

- El desconocimiento de la escritura en Internet. Era un género textual nuevo para ellos. Además, la referencia que tenían era la Wikipedia, que utilizaban con asiduidad, y cuyas características coincidían con la estructuración y formato general del texto expositivo. El tema, esto es, la exposición de lo que se había estudiado en clase de la obra de Unamuno, condicionaba también este estilo expositivo.

- La utilización de hipervínculos, algo novedoso para todos, que les obligó a plantearse a qué debían enlazar. La profesora sugirió que se podían hacer enlaces internos (dentro del mismo wiki) y externos al wiki que estábamos levantando entre todos.

En este último punto hubo que insistir más. Cuando realizaban enlaces internos, no siempre los resultados eran positivos. Por ejemplo, el grupo que trabajaba el Argumento enlazó cada nombre de cada personaje a la página de Personajes. Lo que ocurrió fue que la página de Argumento resultaba ilegible además de inútil en la vinculación, porque se acumularon los hipervínculos siempre dirigidos a la misma página general. Se dieron cuenta finalmente de que un hipervínculo en un personaje 
tiene sentido cuando este personaje tiene una página especial para él solo, pero no cuando es uno más de tantos que configuran la página de Personajes. También enlazaron los títulos de cada capítulo a la página de Estructura. En el foro discuten si es pertinente o no. Finalmente, la revisión en clase les sugirió que debían suprimirlos.

En cuanto a los enlaces externos, es decir aquellos que redirigían a una página de Internet externa al nuestro wiki, también suscitaron dudas. Por ejemplo, una alumna estableció un hipervínculo de Charles Darwin a una fotografía del científico. Al final, corrigió y puso un enlace a la Wikipedia.

Y es que el lenguaje del hipervínculo se tiene también que aprender. Creemos que la mayor parte de los alumnos lo hicieron, aunque, como se ve, todavía el wiki es insuficiente en este aspecto. Un ejemplo de ello es que el grupo del Positivismo enlazó el concepto “Amor y pedagogía” que había escrito en su página a otra del Rincón del vago, que tuvo que suprimirse con posterioridad, como es evidente. La naturaleza del hipertexto $^{1}$ es tan compleja como la misma escritura en Internet, y de él se tiene que aprender la técnica, pero sobre todo aspectos como la pertinencia, la adecuación, la garantía de las fuentes o la implementación del contenido a que vincula.

La función del foro: El foro permitió a los participantes principalmente:

1. Establecer las relaciones entre los participantes.

2. Aclarar aspectos técnicos y formales.

3. Consensuar los diferentes contenido y la estructura del escrito.

4. Fijar una metodología para la toma de acuerdos.

Un ejemplo ilustrativo de cómo se organizan dos grupos diferentes de clases distintas es la figura 1.

Fig. 1: Transcripción 1

\section{ka17 Nov 17, 2008 5:46 am}

Hola. A ver, tendríamos que elegir qué ponemos de cada versión finalmente. Tendríamos que votar cuál nos parece mejor de las dos, y quiatr lo otro,¿no?

mrodena Nov 17, 2008 12:39 pm

Sí, tendriamos que hacer como los grupos de los personajes que han puntuado o por ejemplo de cada tema han dicho que versión les parecia más adecuada! si quereis empezais vosotras opinando, luego nosotras y llegamos a un acuerdo para que poner no?

ka17 Nov 18, 2008 11:03 am

Ok, nuestra opinión (Mireia, Elisabeth, Marta, Carla) es la siguiente: VERSIÓN 1: relación entre el amor y la pedagogía, religión, machismo y educación moral. VERSIÓN 2: conflicto de personalidad, inmortalidad y determinismo. ¿Qué os parece? 
En este ejemplo, los dos grupos que desarrollaban la sección de Temas han elaborado versiones diferentes para el apartado y deciden someter la elección de la definitiva a una votación. Pero igual que sucede con el los grupos de Personajes, se proponen tomar lo mejor de cada uno de los grupos. Posteriormente, en la revisión, pulirían las posibles repeticiones y contradicciones, mejorando los textos iniciales.

Fase de revisión: En el caso de que el agente de la revisión es el mismo grupo, las revisiones se realizan en dos niveles: por una parte, las que atañen al contenido; por otra, las propias de las formas de expresión y ortografía. En las primeras, se tienen en cuenta a su vez tanto la estructura que ha de reflejar la página como la calificación con la que nombran conceptos. Una alumna del grupo de los Personajes propone que deben hacer una introducción de cada uno de los personajes que no repita la que ya tienen escrita del inicio de la página:

Fig. 2: Transcripción 2

marionadorda Jan 22, 2009 9:19 pm

Meri y Susana, os envío lo que colgaria yo en la introducción de los personajes.

Para introducir los personajes es importante hacer una pequeña síntesi de ellos.

marionadorda Jan 22, 2009 9:23 pm

¿Pero Mariona esto iria en la introducción del esquema?

marionadorda Jan 22, 2009 9:26 pm

Si antes, pero más ampliado

$[\ldots]$

(http://amorypedagogia.wikispaces.com/message/view/34+Los+personajes/8549262)

A continuación, este grupo de tres compañeras se intercambian versiones introductorias o desarrolladas de lo que han editado como material base en la página. Como vemos, han quedado de acuerdo en este breve diálogo en cómo estructurarán los personajes: presentación general breve y, antes de analizar cada uno de los personajes, una síntesis de sus características más importantes. Son conscientes, en este caso, de que no pueden empezar de manera abrupta, de que cada personaje equivale a un pequeño capítulo que han de presentar.

En el segundo caso, aquél en que el agente de la revisión es el grupo clase con la mediación de la profesora, esto es, de la revisión colectiva en clase, destacarían una serie de elementos:

- Se proyectaba en clase el wiki, ya que las aulas disponen de conexión a Internet. Algún miembro del grupo cuyo tema se revisaba leía en voz alta lo que salía en pantalla. En la misma lectura, quien leía se apercibía en ocasiones del error, que enmendaba de inmediato (estamos hablando de estudiantes con un conocimiento de 
la lengua bastante amplio). La profesora transcribía los cambios en el mismo wiki, con lo que la sensación de cerrar el texto definitivo era muy positiva. Se preguntaba antes de pulsar el botón “Guardar”, que concluía la revisión.

La lectura que se hace de este punto es que el conocimiento lingüístico es vital en la composición escrita, en la textualización, pero sobre todo en la revisión, cuando se pule el texto y se cierra la versión definitiva.

- En muchos casos, la detección de errores o aspectos mejorables de la expresión, corría a cargo de la profesora, que bien los sugería a partir de inflexiones de voz o con preguntas dirigidas.

En muchas ocasiones el alumnado no se apercibe del error porque le faltan conocimientos lingüísticos o porque no está habituado a ciertas formas de expresión (en castellano, por ejemplo, el uso del relativo “cuyo" suele ser desconocido o resultar ajeno). La profesora en este caso no hace una corrección directa, sino que suscita un interrogante que han de intentar resolver; en caso de que no puedan, ella explica la base teórica lingüística y promueve la solución al problema. A menudo es ella la que tiene que aportarla de manera directa. En todo caso, el problema es detectado en la siguiente ocasión en que surge y corregido por el mismo alumnado.

- Los grupos que trabajaban otros temas diferentes al que se estaba revisando, sugerían mejoras o detectaban contradicciones de contenido que atañían a su campo de estudio. De esa forma, el contenido del wiki se convirtió en algo más sólido gracias a esa mirada contrastiva.

- La mayor parte de propuestas de mejora de expresión o de contenido era aceptada por los miembros de los grupos del tema que se estaba revisando, porque se sabían corresponsables del proyecto común. También porque en otro momento les correspondería a los revisados ser revisadores.

Es curioso comprobar que las correcciones provenientes de los compañeros se aceptan con mayor receptividad. Se supone que vienen de iguales y no suscitan desconfianzas porque se trata de conocimientos que todos comparten; tan sólo ha habido un “despiste” que se modifica sin más problemas. Sin embargo, si es el profesorado quien las hace, se ven como errores, como deficiencias propias, porque el profesor o la profesora evalúan el conocimiento que se tiene de la lengua en este acto. Alumnos de segundo de bachillerato, tan condicionados por el reto de excelencia que tienen delante, lo manifiestan de forma especial. 
- Fue la ocasión para que todos se leyeran y se recordara el conjunto de la obra, dos meses después de haberla leído, con lo que se refrescaron conceptos y se rescató la memoria de los aspectos más débiles.

- Sirvió para revisar conceptos lingüísticos importantes para sus estudios: apropiación de léxico, correlaciones verbales, fórmulas sintácticas, adecuación de las formas de expresión, etc. Resultó especialmente interesante ver todos esos usos de manera práctica, aplicada, y no en simple teoría.

\section{Resultados}

La siguiente parrilla (Cuadro 2) nos puede dar idea de la interacción llevada a cabo en cada una de las páginas del wiki. Siempre hay intervenciones de la profesora, tanto en los foros de discusión como en las revisiones. Hay que tener en cuenta que, como mínimo, una de las revisiones las realizó la profesora, pero corresponde a la revisión global realizada en la clase con la aportación de todos.

Cuadro 2: Interacción de la páginas del wiki

\begin{tabular}{|l|l|l|l|l|l|}
\hline \multicolumn{1}{|c|}{ Temas } & Autores & $\mathbf{N}^{\mathbf{0}}$ grupos & $\mathbf{N}^{\mathbf{0}}$ discusiones & \multicolumn{1}{c|}{$\begin{array}{c}\text { Utilización } \\
\text { chat }\end{array}$} & \multicolumn{1}{|c|}{$\mathbf{N}^{\mathbf{0}}$ revisiones } \\
\hline $\begin{array}{l}\text { 1. Introducción al } \\
\text { pensamiento de la época }\end{array}$ & 1 & 1 & 0 & No & 1 (profesora) \\
\hline Evolucionismo & 3 & 1 & 2 & No & 12 (6 profesora) \\
\hline El krausismo y la ILE & 3 & 1 & 1 & No & 13 (2 profesora) \\
\hline El positivismo & 3 & 1 & 17 & Ś́ & $66(2$ profesora $)$ \\
\hline La Generación del 98 & 3 & 1 & 0 & No & 21 (2 profesora) \\
\hline 2. Miguel de Unamuno & 6 & 2 & 2 & No & 33 (3 profesora) \\
\hline 3. La obra & 1 & 1 & 0 & No & 8 (6 profesora) \\
\hline Argumento & 6 & 2 & 24 & No & 27 (5 profesora) \\
\hline Temas & 7 & 2 & 15 & Sí & 48 (2 profesora) \\
\hline La estructura & 6 & 2 & 2 & No & 13 (5 profesora) \\
\hline Los personajes & 11 & 3 & 60 & No & 40 (5 profesora) \\
\hline
\end{tabular}

¿Qué ideas podemos extraer de este esquema? Las resumimos en los siguientes puntos:

1. Sólo hay dos temas redactados cada uno de ellos por una sola persona y que muestran el índice de autonomía que poseen. Mientras que la persona encargada de la introducción a la época tiene las cosas claras y sólo necesita la corrección global del grupo clase, la persona que se encargó de la introducción a la obra es menos autónoma y requiere de 8 revisiones: 2 personales, 5 de la profesora y 1 global del conjunto de la clase.

2. Hay grupos que no utilizan el foro de discusión como herramienta de interacción asíncrona (La Generación del 98) o lo utilizan muy poco (Evolucionismo, El 
Krausismo y la ILE, Miguel de Unamuno, La estructura). Cuando lo utilizan, le sacan poco provecho, ya que no interactúan en él. Plantean dudas que no se contestan. A pesar de todo, son grupos que pertenecen a la misma clase y quedan allí para discutir cuestiones relativas al contenido y a la coordinación del equipo. Acostumbran a ser grupos formados por alumnos de rendimiento bajo, con escasas excepciones, al menos en el caso que planteamos. La confección de grupos de trabajo siempre es problemática, ya que depende de los objetivos que nos propongamos, de la motivación intrínseca del mismo alumnado y de la extrínseca que proporciona la misma actividad o los compañeros con los que se comparta el objetivo.

3. Los grupos con más interacción son también los más autónomos (El Positivismo, Argumento, Temas y Personajes). El número de sus discusiones es elevado y, en cambio, la profesora interviene poco en las revisiones. Sólo acuden a ella cuando entre los componentes de los grupos no pueden ponerse de acuerdo o no comparten el mismo recuerdo de la tarea. Las revisiones que muestra el historial de cada página marcan modificaciones en los siguientes aspectos ${ }^{2}$ :

- Ortografía: tildes, comillas, cursivas...

- Tipografía: tipo de guiones de enumeración, errores en la escritura de palabras, espacios antes o detrás de las palabras, formato de los títulos...

- Morfología: concordancias de género, uso de preposiciones o de conectores, cambios de tiempos verbales que guarden la correlación correcta. Ver figura 3.

Fig. 3: Ejemplo 1 de Historial del wiki

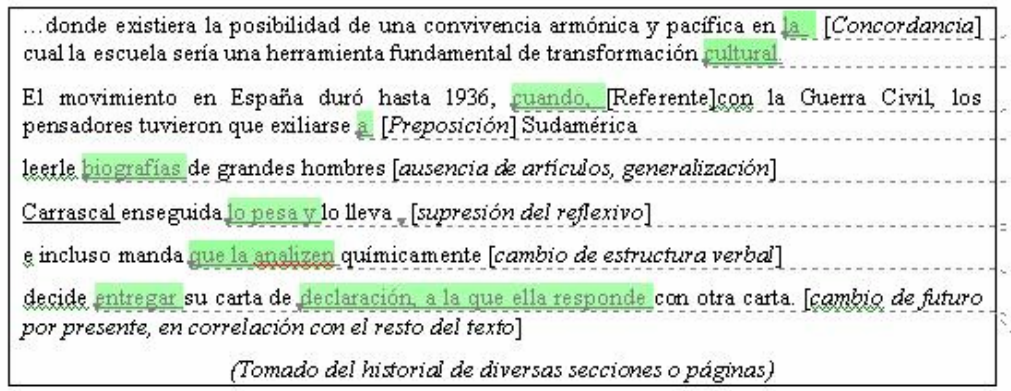

\begin{tabular}{|c|}
\hline Eliminado: el \\
\hline Eliminado: cultural \\
\hline Eliminado: que \\
\hline Eliminado: en \\
\hline Eliminado: la biografia \\
\hline Eliminado: se \\
\hline Eliminado: a la balanza \\
\hline Eliminado: a malizarla \\
\hline $\begin{array}{l}\text { Eliminado: entregar, más } \\
\text { adelante. }\end{array}$ \\
\hline $\begin{array}{l}\text { Eliminado: declaración y } \\
\text { ésta le corresponderá }\end{array}$ \\
\hline
\end{tabular}


- Sintaxis: cambio de orden de las extensiones de las oraciones para que resulten más claras, supresión de elementos redundantes, cambios en estructuras sintácticas para evitar circunloquios, supresión o modificación de conectores que sean más adecuados a las intenciones del texto, implementación de elementos clarificadores del significado. Ver figura 4.

Fig. 4: Ejemplo 2 de Historial del wiki
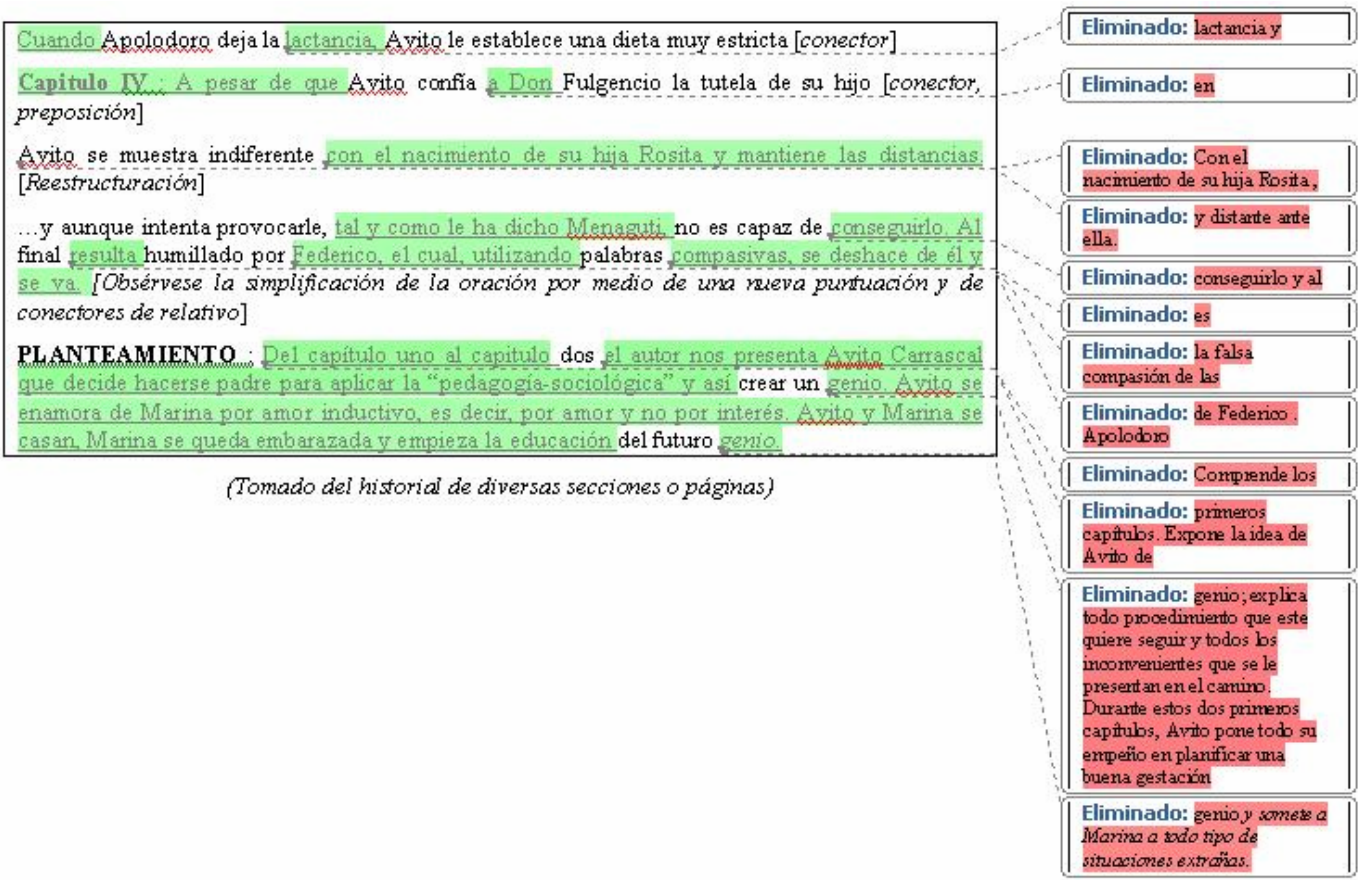

En este último caso vemos que la ordenación de la oración es global: además de simplificar la oración anterior, simplifica también el contenido, evitando los matices secundarios.

- Léxico: se buscan las palabras más apropiadas al contexto en que se publica o se modifican palabras que no son adecuadas. O se simplifican expresiones innecesarias. Por ejemplo, ver figura 5.

Fig. 5: Ejemplo 3 de Historial del wiki

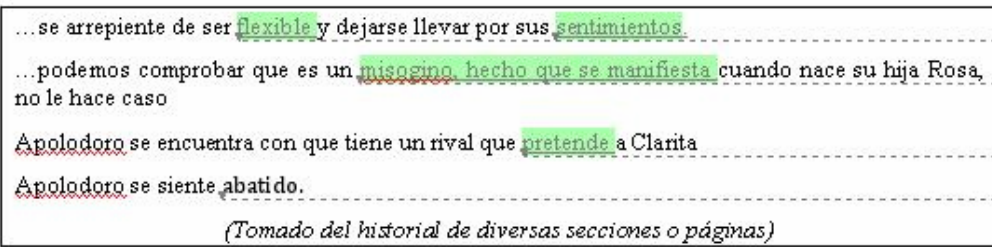

(Tomado del historial de diversas secciones o páginas)

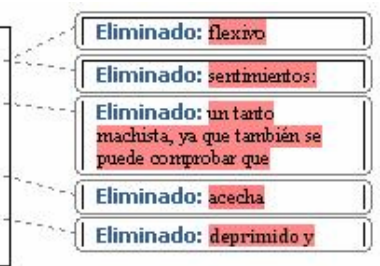

- Estructura: cambios en la estructura del contenido para que quede más ordenado. 
- Contenido: implementación de textos que completan el contenido cuando éste es deficiente o corrección de errores de concepto.

- Enlaces: añaden o suprimen según los acuerdos tomados o las sugerencias recibidas.

- Netgrafía (término acuñado por una de las compañeras de los bachilleratos que tuvo rápida acogida por parte de todos): se completa paulatinamente a medida que descubren nuevas fuentes.

\section{Conclusiones}

Podemos resumir las conclusiones extraídas del trabajo en las siguientes reflexiones acerca de las dificultades y aciertos del trabajo colaborativo en el wiki.

En primer lugar, las hojas de autoevaluación y evaluación mutua, como elemento de triangulación de la validez del trabajo, permitieron detectar la visión del alumnado respecto del trabajo colaborativo a partir de este entorno tecnológico:

- Los grupos sólo desarrollaban una parte del libro, con lo que se perdían la profundización del resto. En contra, otra alumna se muestra satisfecha porque, además de apreciar todo lo que se puede aprender con una sola lectura, se aprende más todavía leyendo los trabajos de los demás.

La interpretación que debemos hacer de esta intervención es que no hay un criterio compartido respecto al aprendizaje individual de los contenidos elaborados. Se aprende mucho de un concepto o tema, el que se ha desarrollado, pero no tanto de los temas desarrollados por otros grupos, excepción hecha de algún caso.

- Algunos de los alumnos no podían acceder al wiki, con lo que dependían de otras personas, a veces del otro grupo, para introducir sus trabajos.

- Dificultades puntuales a la hora de coordinarse con los compañeros y compañeras del otro grupo. Era esperable que el foro del wiki sirviera a tal propósito, pero no se ha acabado de consolidar como elemento de coordinación en algún grupo de trabajo. Por contra,

- Otros grupos sí habían aprendido a organizarse. Un grupo especialmente nos muestra una de las claves: se repartieron los capítulos del argumento que debían desarrollar, pero después quedan a través del foro del wiki para retocárselos. Alguna alumna muestra su descontento a la profesora porque había hecho un capítulo muy extenso y se lo habían recortado los demás. El trabajo colaborativo no lo tiene muy 
asumido. No obstante, sus aportaciones, según el historial del wiki, no acaban de demostrar la razón de su descontento.

- Reconocen, en general, que “la cooperación nos ayuda a aprobar”. Que han podido ponerse "de acuerdo y relacionar la información que cada uno había buscado"; es decir, a sintetizar y resumir; en otras palabras, a "transformar la información que encontraba con mis palabras y expresiones”. Que han aprendido de los errores y a “Estructurar mi trabajo en grupo y saber juntar diversas informaciones”.

En definitiva, el trabajo cooperativo y la interacción los consideran positivos en tanto aprenden y, al aprender, consiguen mejores resultados y, por tanto, mejores calificaciones. Además, se hacen conscientes de que la apropiación de conocimientos sólo es posible cuando uno es capaz de expresar con palabras propias aquello que sabe. Se trata de cambiar el sistema reproductivo de enseñanza por un sistema activo de producción.

- Alguna acusación del tipo "los otros no hacen nada y nosotros hemos tenido que hacerlo todo". Sin fundamento, por otra parte, dado que los registros de los foros constatan el trabajo de cada grupo. Se trata por lo general de alumnos y alumnas muy exigentes con su propio trabajo.

Para completar en cierta medida los resultados obtenidos en esta actividad, quedaría una última consideración que hacer, y que se resumen en las dificultades principales detectadas en el wiki como tecnología y, por otra parte, en las dificultades y aciertos detectados en el trabajo cooperativo.

Los aciertos del wiki, tanto para el alumnado como para la profesora son evidentes y creo que han quedado más o menos claros anteriormente. Ahora bien, también entrañó dificultades sobre las que se tiene que advertir. En primer lugar, se trata de una herramienta que no permite la simultaneidad de acciones, de manera que cuando dos alumnos coincidían en el tiempo y en el espacio, el trabajo de uno “machacaba” el trabajo de otro, con el consiguiente enfado del segundo.

En segundo lugar, hubo también dificultades técnicas muy condicionadoras, como el hecho de que no dispone de un procesador de texto avanzado y determinada tipografía no la admitía o no guardaba reconocía cambios de letra y/o tamaño. Un problema menor, aunque los alumnos se mostraban muy contrariados con él, fue el idioma. Todo está en inglés, y en ocasiones pensaban que no habían publicado bien porque no entendían con suficiente claridad la lengua. 
Otra complicación importante fue el que no todos los alumnos pudieron entrar en el wiki como usuarios del mismo. Lo compensaron accediendo con el nombre de usuario y la contraseña de otro compañero o de otra compañera e identificándose en el foro de discusión con su propio nombre, pero no dejaba de ser incómodo y en cierta medida farragoso. Hubo momentos en que la prontitud de los mensajes del foro les hacía olvidar identificarse y otra compañera les urgía a hacerlo porque desconocía con quién se estaba escribiendo.

Por ese mismo motivo, la profesora sólo pudo valorar el trabajo realizado por aquellos alumnos que se identificaban o que entraban en el wiki. Quedaban algunos alumnos o alumnas pendientes de evaluación continua porque había sido imposible su entrada en la herramienta de internet. Para solucionar este problema, se podría hacer un tutorial claro y visualizador de los pasos que hay que seguir para ser usuario editor de un wiki.

Además, se tiene que explicar con claridad el lenguaje hipertextual y las funciones del hipervínculo antes de editar un wiki, porque, aunque se trata de un procedimiento muy familiar para nuestro alumnado, lo es en tanto que son lectores de Internet, pero no como escritores de Internet. En la sociedad actual cada vez son más necesarios estos procedimientos y su manejo. Sólo este tema daría para un trabajo de investigación. No es igual leer en Internet que escribir en ella. Tampoco se ha de suponer que si se sabe leer correctamente el hipervínculo, descartando los peligros de lectura discontinua que posee, se ha de saber, en consecuencia, escribir correctamente con los hipervínculos adecuados.

En cuanto a las dificultades detectadas en el trabajo cooperativo son varios. En primer lugar, ponerse de acuerdo dos grupos pertenecientes a dos clases diferentes no es fácil. En esta ocasión los grupos de Temas y Personajes sufre, pero acaban coordinándose muy bien. Por otra parte, escoger versiones realizadas por los diferentes grupos para publicarlas en el wiki suscita controversias: llegan fácilmente a un acuerdo democrático que no hiere susceptibilidades a partir del sistema de votaciones, aunque la versión finalmente escogida puede ser modificada por otros componentes del grupo. En tercer lugar, provoca egos maltrechos: “yo he trabajado más y no lo has visto”. Se trata de la única persona que mostró nerviosismo en el foro. Las relaciones con su grupo acabaron viciándose en la misma aula. 
Podemos añadir a todo ello que, en general, necesitan más tiempo para la realización del trabajo, más negociación; este tipo de trabajo colaborativo suele provocar, por tanto, más estrés.

Si la tecnología pudiera combinar el método asíncrono del wiki con otro sincrónico, se facilitaría el trabajo cooperativo y la toma de acuerdos inmediata.

No obstante, el trabajo cooperativo ha tenido aciertos en esta actividad de aula. A saber: el alumnado aprende a negociar, a buscar la fórmula más justa que satisfaga a todos al mismo tiempo que consolidan contenidos. Esta negociación enriquece la perspectiva que del contenido tienen los componentes de los grupos y se ayudan a plasmarlo en el wiki, herramienta de contenidos de Internet. También ha sido importante el compartir una base de conocimientos previos que se facilitó en clase, aunque, con todo, no deja de haber desigualdades entre los alumnos, que ni trabajan de la misma manera ni con la misma intensidad ni con la misma comprensión del contenido. El wiki facilita la distribución de tareas en función de esas dificultades bien de manera planificada, bien de forma espontánea. La primera parte de la profesora al proponer temas a determinados alumnos o grupos. La segunda se realiza entre los componentes de los grupos espontáneamente cuando otorgan el papel de organizador o de coordinador o de revisor a alguno de ellos.

En definitiva, consideramos que esta herramienta de Internet permite a alumnado y profesorado encarar, a partir de una nueva estrategia de enseñanza y aprendizaje, nuevos retos de la educación. En lo que compete al tema central de este trabajo, la escritura colaborativa, creemos firmemente que es un elemento a potenciar en las clases. No nos hemos podido detener en el proceso de textualización porque no se manifiesta en el wiki y tampoco era nuestro propósito. Sí se puede elaborar un análisis más exhaustivo en el proceso de revisión que éste que se presenta, ya que se dispone de foros e historial de cambios muy valiosos para el docente, quien debe valorar el trabajo aportado por cada uno de sus estudiantes. Un estudio más profundo mostraría una interesante reflexión metalingüística en este proceso.

La intención del trabajo era mostrar las ventajas de la escritura colaborativa en entornos tecnológicos, por la escritura en sí como elemento de construcción de conocimiento si está ligada a temas académicos, pero sobre todo por ser colaborativa, por fomentar el trabajo cooperativo en interacción entre los estudiantes, ya que desarrollan estrategias interesantes de coordinación y de revisión dignas de tener en cuenta en trabajos posteriores. 


\section{Referencias bibliográficas}

Bustos Sánchez, A. (2009): "Escritura colaborativa en línea. Un estudio preliminar orientado al análisis del proceso de co-autoría” [en línea]. En RIED - Revista Iberoamericana de Educación a Distancia, Volumen 12, N² 2, 2009, ISSN: 1138 $-$

http://www.utpl.edu.ec/ried/images/pdfs/vol12N2/escrituracolaborativa.pdf 2783. [Consultado el 3 de enero de 2010].

Camps, A. (1994): L'ensenyament de la composició escrita, Barcanova.

Coll, C. (2005): “Lectura y alfabetismo en la sociedad de la información” [en línea]. En UOC papers, Revista de la societat del coneixement, núm. 1. ISSN: 1885-1541. http://www.uoc.edu/uocpapers/1/dt/esp/coll.pdf [Consultado el 5 de enero de 2010]

Esteve, O. (2002): "La interacción en el aula desde el punto de vista de la coconstrucción de conocimientos: un planteamiento didáctico”, en La lengua, vehículo cultural multidisciplinar, coord. por María Sagrario Salaberri Ramiro, ISBN 84-369-3602-7, págs. 61-82.

Lave, J., Wegner, E. (1991): Situated Learning and Peripheral Participation, Cambridge, Cambridge University Press.

Paredes, M., Fernández, I., Ortega, M., Velázquez, J. A. (2005): “Aprendizaje colaborativo de la escritura mediante Organización de Contenidos y Tareas” [en línea]. En Revista Iberoamericana de Informática Educativa, n 1, Enero 2005 (21-30).

http://chico.infr.uclm.es/eCLUB/articulos/ArticulosRevistasInternacionales/ARI20.pdf [Consultado el 4 de enero de 2009)

Scardamaglia, M. y Bereiter, C. (1994): “Computer support for knowledge-building communities” [en línea]. The Journal of the Learning Sciences, 3(3), 265-283. En http://carbon.ucdenver.edu/ bwilson/building.html [Consultado el 17 de enero de 2011]

Vigotsky, L. (1995) Pensamiento y lenguaje. Buenos Aires: La Pléyade.

\footnotetext{
1 "Hipertexto: qué es y cómo utilizarlo para escribir en medios electrónicos" (5 de julio de 2003). Consultado en http://www.eduteka.org/Hipertexto1.php el 7 de enero de 2010.

2 Los subrayados en verde marcan lo que se añade, lo que se corrige, mientras que los subrayados rojos evidencian lo que se suprime. Todas las modificaciones están señaladas por la persona que las realiza, y la hora y el número de orden que les corresponde en el total de modificaciones producidas.
}

\footnotetext{
Referencias de la autora:

Inma López es catedrática de Lengua y Literatura Española en Educación Secundaria, Máster de Investigación en Didáctica de la Lengua y la Literatura de la UAB y formadora del ICE de la misma Universidad. Formada en la metodología de la práctica reflexiva. Ha colaborado el Àrea de Programes de Formació del Departament d'Educació en diversos temas y protocolos relacionados con el asesoramiento en centros, el trabajo por competencias y la programación por competencias. Forma parte del grupo GREAL de la UAB. Tiene diversas publicaciones relacionadas con la lectura en Secundaria y el trabajo por competencias en el área lingüística, tales como: Llengua catalana i literatura, Llengua castellana $i$ literatura, Segon cicle d'ESO (Ed. ICE UAB ISBN: 84-89489-53-X, de autoría compartida), Llegir per aprendre. Una proposta per treballar les lectures a l'ESO (Sèrie Eines i Estratègies, 16. Els llibres de la UAB. ISBN: 84-89489-71-8 de autoría compartida) y La palabra compartida. La competencia comunicativa en el aula (Octaedro. ISBN: 978-84-9921-006-3, de autoría compartida). Mantiene diversos blogs de clase y un aula de recursos y bibliografía en Espacio de Lengua: http://phobos.xtec.cat/ilopez15/.

Email: ilopez62@gmail.com
} 